\title{
Homology operations in the topological cyclic homology of a point
}

\author{
HÅKON SCHAD BERGSAKER \\ JOHN ROGNES
}

\begin{abstract}
We consider the commutative $\mathbb{S}$-algebra given by the topological cyclic homology of a point. The induced Dyer-Lashof operations in $\bmod p$ homology are shown to be nontrivial for $p=2$, and an explicit formula is given. As a part of the calculation, we are led to compare the fixed point spectrum $\mathbb{S}^{G}$ of the sphere spectrum and the algebraic $K$-theory spectrum of finite $G$-sets, as structured ring spectra.
\end{abstract}

55S12, 55P43; 19D55, 55P92, 19D10

\section{Introduction}

Let $A(\star)=K(\mathbb{S})$ denote Waldhausen's algebraic $K$-theory of a point [23]. It is a commutative $\mathbb{S}$-algebra, in the sense of Elmendorf, Kriz, Mandell and May [7], and the algebraic $K$-theory $A(X)$ of any space $X$, or more generally the algebraic $K$-theory $K(R)$ of any $\mathbb{S}$-algebra $R$, is a module spectrum over it. Hence it makes sense to carefully study the commutative $\mathbb{S}$-algebra structure of $A(\star)$, or equivalently its structure as an $E_{\infty}$ ring spectrum. To the eyes of $\bmod p$ homology, the primary incarnation of this structure is the Pontryagin algebra structure on $H_{*}(A(\star))$, together with the multiplicative Dyer-Lashof operations $Q^{i}: H_{*}(A(\star)) \rightarrow H_{*+i}(A(\star))$, as defined by Bruner, May, McClure and Steinberger [2]. Here and elsewhere we write $H_{*}(E)$ for the $\bmod p$ homology $H_{*}\left(E ; \mathbb{F}_{p}\right)$ of a spectrum $E$.

The additive structure of $H_{*}(A(\star))$ is known for $p=2$ and for $p$ an odd regular prime, by the second author's papers [18; 19], but at present the Pontryagin product and Dyer-Lashof operations are not known for this $E_{\infty}$ ring spectrum. There is, however, a very good approximation to Waldhausen's algebraic $K$-theory, given by the cyclotomic trace map to the topological cyclic homology of Bökstedt, Hsiang and Madsen [1]. This is a natural map trc: $K(R) \rightarrow \mathrm{TC}(R ; p)$, which we write as trc: $A(\star) \rightarrow \mathrm{TC}(\star ; p)$ in the special case when $R=\mathbb{S}$, where $\mathrm{TC}(\star ; p)=\mathrm{TC}(\mathbb{S} ; p)$ is the topological cyclic 
homology of a point. By a theorem of Dundas [5], there is a homotopy cartesian square

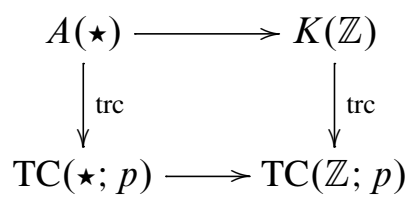

(after $p$-adic completion) of commutative $\mathbb{S}$-algebras (see Geisser and Hesselholt [9, Section 6]), and this square is the basis for our additive understanding of $H_{*}(A(\star))$.

We are therefore led to study the commutative $\mathbb{S}$-algebra structure of $\mathrm{TC}(\star ; p)$, including the Pontryagin algebra structure and the Dyer-Lashof operations on its mod $p$ homology. Like in the case of algebraic $K$-theory, the topological cyclic homology $\mathrm{TC}(X ; p)$ of any space $X$, and more generally the topological cyclic homology $\mathrm{TC}(R ; p)$ of any $\mathbb{S}$-algebra $R$, is a module spectrum over $\mathrm{TC}(\star ; p)$, and this provides a second motivation for the study of $\mathrm{TC}(\star ; p)$. In the present paper, we determine the Dyer-Lashof operations in $H_{*}(\mathrm{TC}(\star ; p))$ in the case when $p=2$, as explained in Theorem 0.2 and Corollary 0.3 below.

A third motivation stems from ideas of Jack Morava [17], to the effect that there may be a spectral enrichment of the algebro-geometric category of mixed Tate motives, given by $A$-theoretic (see Williams [24]) or TC-theoretic (see Dundas and Østvær [6]) correspondences, followed by stabilization. The trace map $A(\star) \rightarrow \mathrm{TC}(\star ; p) \rightarrow \mathrm{THH}(\star)=\mathbb{S}$ defines a fiber functor to the category of $\mathbb{S}$-modules, with Tannakian automorphism group realized through its Hopf algebra of functions, which will be of the form $\mathbb{S} \wedge_{A(\star)} \mathbb{S}$ or $\mathbb{S} \wedge_{\mathrm{TC}(\star ; p)} \mathbb{S}$. Rationally, this is well compatible with Deligne's results on the Tannakian group of mixed Tate motives over the integers [4]. A calculational analysis of the commutative $\mathbb{S}$-algebras $\mathbb{S} \wedge_{A(\star)} \mathbb{S}$ or $\mathbb{S} \wedge_{\mathrm{TC}(\star ; p)} \mathbb{S}$ clearly depends heavily on a proper understanding of the commutative $\mathbb{S}$-algebra structures of $A(\star)$ and $\mathrm{TC}(\star ; p)$.

Let $\mathbb{T}$ be the circle group and let $C_{p^{n}} \subset \mathbb{T}$ be the (cyclic) subgroup of order $p^{n}$. The spectrum $\mathrm{TC}(\star ; p)$ is defined as the homotopy inverse limit of a diagram

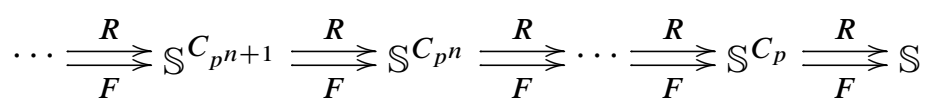

of $E_{\infty}$ ring spectra, where $\mathbb{S}^{C_{p^{n}}}$ denotes the $C_{p^{n}}$-fixed points of the $\mathbb{T}$-equivariant sphere spectrum, the maps labeled $R$ are restriction maps, and the maps labeled $F$ are Frobenius maps. See Bökstedt, Hsiang and Madsen [1] or Hesselholt and Madsen [10] for the construction of these maps. Similarly, let $\mathrm{TC}^{(1)}(\star ; p)$ denote the homotopy 
limit of the subdiagram

$$
\mathbb{S} C_{p} \underset{F}{\stackrel{R}{\longrightarrow}} \mathbb{S},
$$

that is, the homotopy equalizer of $R$ and $F$. The canonical maps

$$
\mathrm{TC}(\star ; p) \stackrel{f_{1}}{\longrightarrow} \mathrm{TC}^{(1)}(\star ; p) \stackrel{g_{1}}{\longrightarrow} \mathbb{S} C_{p}
$$

are then maps of $E_{\infty}$ ring spectra.

The unit $\eta: \mathbb{S} \rightarrow \mathrm{TC}(\star ; p)$ and the restriction $R: \mathbb{S} C_{p} \rightarrow \mathbb{S}$ let us split off a copy of $\mathbb{S}$ from each term in (0-3). Let $\mathbb{C} P_{-1}^{\infty}$ be the Thom spectrum of the negative tautological complex line bundle $-\gamma_{\mathbb{C}}^{1}$ over $\mathbb{C} P^{\infty}$. Its suspension $\Sigma \mathbb{C} P_{-1}^{\infty}$ is equivalent to the homotopy fiber of the dimension-shifting $\mathbb{T}$-transfer map $t_{\mathbb{T}}: \Sigma^{\infty} \Sigma\left(\mathbb{C} P_{+}^{\infty}\right) \rightarrow \mathbb{S}$; see Knapp [13, 2.9] or Lemma 1.1 below. We define the spectrum $L_{-1}^{\infty}$ to be the homotopy fiber of the $C_{p}$-transfer $t_{p}: \Sigma^{\infty}\left(B C_{p}\right)_{+} \rightarrow \mathbb{S}$. For $p=2$, there is an equivalence $L_{-1}^{\infty} \simeq \mathbb{R} P_{-1}^{\infty}$, where $\mathbb{R} P_{-1}^{\infty}$ is the Thom spectrum of the negative tautological real line bundle $-\gamma_{\mathbb{R}}^{1}$ over $\mathbb{R} P^{\infty}$. The mod $p$ homology groups of these spectra are well known:

$$
\begin{aligned}
H_{*}\left(\Sigma \mathbb{C} P_{-1}^{\infty}\right) & \cong \mathbb{F}_{p}\left\{\Sigma \beta_{k} \mid k \geq-1\right\} \\
H_{*}\left(L_{-1}^{\infty}\right) & \cong \mathbb{F}_{p}\left\{\alpha_{k} \mid k \geq-1\right\} \\
H_{*}\left(\Sigma^{\infty}\left(B C_{p}\right)_{+}\right) & \cong \mathbb{F}_{p}\left\{\alpha_{k} \mid k \geq 0\right\}
\end{aligned}
$$

Here $\Sigma \beta_{k}$ has degree $2 k+1$ and $\alpha_{k}$ has degree $k$.

Lemma 0.1 After $p$-completion, diagram (0-3) is homotopy equivalent to a diagram

$$
\mathbb{S} \vee \Sigma \mathbb{C} P_{-1}^{\infty} \stackrel{1 \vee f}{\longrightarrow} \mathbb{S} \vee L_{-1}^{\infty} \stackrel{1 \vee g}{\longrightarrow} \mathbb{S} \vee \Sigma^{\infty}\left(B C_{p}\right)_{+} .
$$

In particular, the Pontryagin product on $H_{*}(\mathrm{TC}(\star ; p))$ is trivial.

Applying homology gives a sequence

$$
H_{*}(\mathbb{S}) \oplus H_{*}\left(\Sigma \mathbb{C} P_{-1}^{\infty}\right) \stackrel{1 \oplus f_{*}}{\longrightarrow} H_{*}(\mathbb{S}) \oplus H_{*}\left(L_{-1}^{\infty}\right) \stackrel{1 \oplus g_{*}}{\longrightarrow} H_{*}(\mathbb{S}) \oplus H_{*}\left(\Sigma^{\infty}\left(B C_{p}\right)_{+}\right) .
$$

Here $f_{*}$ sends $\Sigma \beta_{k}$ to $\alpha_{2 k+1}$ for $k \geq-1$, and $g_{*}$ is the identity on $\alpha_{k}$ for $k \geq 0$, while $\alpha_{-1}$ maps to zero.

We now state our main result, which concerns the Dyer-Lashof operations in the mod $p$ spectrum homology $H_{*}(\mathrm{TC}(\star ; p))$ for $p=2$. The calculations will be done in the auxiliary $E_{\infty}$ ring spectra $\mathbb{S}^{C_{2}}$ and $\mathrm{TC}^{(1)}(\star ; 2)$. 
Theorem 0.2 The Dyer-Lashof operations $Q^{i}$ in $H_{*}\left(\mathrm{TC}^{(1)}(\star ; 2)\right)$ and $H_{*}\left(\mathbb{S}^{C_{2}}\right)$ are given by the formula

$$
Q^{i}\left(\alpha_{j}\right)=\left(\begin{array}{c}
2^{N}+i-1 \\
2^{N}+j
\end{array}\right) \alpha_{i+j}
$$

where $j \geq-1$ and $i$ is any integer, and $N$ is sufficiently large.

Corollary 0.3 The Dyer-Lashof operations $Q^{2 i}$ in $H_{*}(\mathrm{TC}(\star ; 2))$ are given by the formula

$$
Q^{2 i}\left(\Sigma \beta_{j}\right)=\left(\begin{array}{c}
2^{N}+i-1 \\
2^{N}+j
\end{array}\right) \Sigma \beta_{i+j}
$$

where $j \geq-1$ and $i$ is any integer, and $N$ is sufficiently large. The operations $Q^{2 i+1}$ are all zero for degree reasons.

Note that the binomial coefficients used in the theorem and corollary can be evaluated to

$$
\left(\begin{array}{cl}
2^{N}+i-1 \\
2^{N}+j
\end{array}\right) \equiv \begin{cases}\left(\begin{array}{c}
i-1 \\
j
\end{array}\right) & \text { for } i>j \geq 0 \\
1 & \text { for }(i, j)=(0,-1) \\
0 & \text { otherwise }\end{cases}
$$

modulo 2, for all sufficiently large $N$. In particular $Q^{0}\left(\alpha_{-1}\right)=\alpha_{-1}$ and $Q^{0}\left(\Sigma \beta_{-1}\right)=$ $\Sigma \beta_{-1}$.

We prove Lemma 0.1 in Section 1 and Theorem 0.2 in Section 3, after a homological comparison of $E_{\infty}$ ring structures in Section 2. Corollary 0.3 follows immediately from the lemma and the theorem.

\section{Topological cyclic homology of a point}

In this preliminary section we review the calculation of $\mathrm{TC}(\star ; p)$ from Bökstedt, Hsiang and Madsen $[1,5.17]$, in order to describe the map $f_{1}$ to $\operatorname{TC}^{(1)}(\star ; p)$.

For each $n \geq 1$ the Segal-tom Dieck splitting tells us that the norm-restriction homotopy cofiber sequence

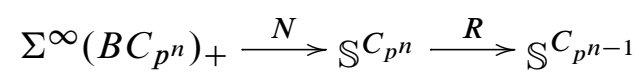

is canonically split. The homotopy limit $\operatorname{TR}(\star ; p)=\operatorname{holim}_{n, R} \mathbb{S} C_{p^{n}}$ of the $R$-maps in (0-1) thus factors as $\operatorname{TR}(\star ; p) \simeq \prod_{n \geq 0} \Sigma^{\infty}\left(B C_{p^{n}}\right)_{+}$. Let

$$
\operatorname{pr}_{n}: \operatorname{TR}(\star ; p) \rightarrow \Sigma^{\infty}\left(B C_{p^{n}}\right)_{+}
$$


denote the $n$-th projection, and let $\widetilde{\mathrm{TR}}(\star ; p) \simeq \prod_{n \geq 1} \Sigma^{\infty}\left(B C_{p^{n}}\right)+$ be the homotopy fiber of $\mathrm{pr}_{0}$. There is a vertical map of horizontal homotopy fiber sequences

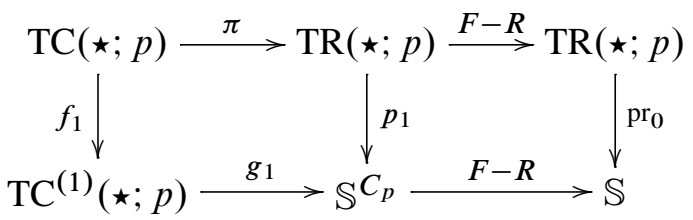

and the augmentation $\mathrm{TC}(\star ; p) \rightarrow \mathbb{S}$ factors as $R \circ p_{1} \circ \pi=\mathrm{pr}_{0} \circ \pi$. Replacing the left hand square by the homotopy fibers of the augmentations to $\mathbb{S}$, we get a second vertical map of horizontal homotopy fiber sequences

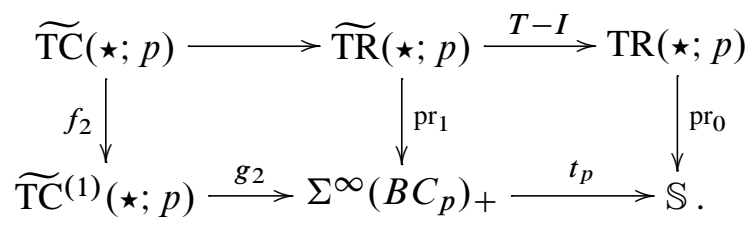

In the upper row we have used that $F-R$ restricted along the inclusion $I: \widetilde{T R}(\star ; p) \rightarrow$ $\mathrm{TR}(\star ; p)$ is homotopic to $T-I$, where $T$ is the product of the $C_{p}$-transfer maps $\Sigma^{\infty}\left(B C_{p^{n}}\right)_{+} \rightarrow \Sigma^{\infty}\left(B C_{p^{n-1}}\right)_{+}$for all $n \geq 1$. See Bökstedt, Hsiang and Madsen [1, (5.18)]. In the lower row we have used that $F-R$ restricted along $N: \Sigma^{\infty}\left(B C_{p}\right)_{+} \rightarrow$ ${ }_{S} C_{p}$ is homotopic to the $C_{p}$-transfer map $t_{p}$.

There is a third vertical map of horizontal homotopy fiber sequences

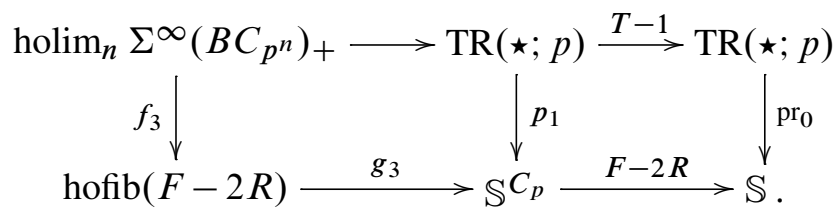

Replacing its left hand square by the homotopy fibers of the augmentations to $\mathbb{S}$, we also recover diagram (1-2).

Lemma 1.1 There are equivalences

$$
\widetilde{\mathrm{TC}}(\star ; p) \simeq \operatorname{hofib}\left(t_{\mathbb{T}}: \Sigma^{\infty} \Sigma\left(\mathbb{C} P_{+}^{\infty}\right) \rightarrow \mathbb{S}\right) \simeq \Sigma \mathbb{C} P_{-1}^{\infty}
$$

(after $p$-completion) and

$$
\widetilde{\mathrm{TC}}^{(1)}(\star ; p) \simeq \operatorname{hofib}\left(t_{p}: \Sigma^{\infty}\left(B C_{p}\right)_{+} \rightarrow \mathbb{S}\right)=L_{-1}^{\infty} .
$$

When $p=2, L_{-1}^{\infty} \simeq \mathbb{R} P_{-1}^{\infty}$. 
Proof The dimension-shifting $\mathbb{T}$-transfer maps for the bundles $B C_{p^{n}} \rightarrow B \mathbb{T}$ induce an equivalence $\Sigma^{\infty} \Sigma\left(\mathbb{C} P_{+}^{\infty}\right) \simeq \operatorname{holim}_{n} \Sigma^{\infty}\left(B C_{p^{n}}\right)_{+}$after $p$-completion [1, 5.15]. The augmentation $\operatorname{holim}_{n} \Sigma^{\infty}\left(B C_{p^{n}}\right)_{+} \rightarrow \mathbb{S}$ then gets identified with $t_{\mathbb{T}}$, which implies the first claim.

There is a $\mathbb{T}$-equivariant homotopy cofiber sequence $S^{0} \stackrel{z}{\rightarrow} S^{\mathbb{C}} \stackrel{t}{\rightarrow} \mathbb{T}_{+} \wedge S^{1}$, where $z$ is the zero-inclusion. The right hand map $t$ is the Pontryagin-Thom collapse associated to the standard embedding $\mathbb{T} \subset \mathbb{C}$, as in Lewis, May and Steinberger [14, II.5.1]. The dimension-shifting $\mathbb{T}$-transfer $t_{\mathbb{T}}: \Sigma^{\infty} \Sigma\left(\mathbb{C} P_{+}^{\infty}\right) \rightarrow \mathbb{S}$ for $E \mathbb{T} \rightarrow B \mathbb{T}$ is constructed as the balanced smash product

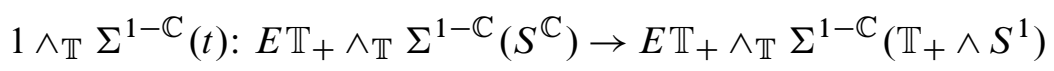

(see Lewis, May and Steinberger [14, II.7.5]). Hence its homotopy fiber is $E \mathbb{T}_{+} \wedge_{\mathbb{T}}$ $\Sigma^{1-\mathbb{C}}\left(S^{0}\right) \cong \Sigma \mathbb{C} P_{-1}^{\infty}$.

The proof that $\mathbb{R} P_{-1}^{\infty}$ is the homotopy fiber of $t_{p}$ for $p=2$ is essentially the same.

Proof of Lemma 0.1 Under the identifications of Lemma 1.1, the maps $f: \Sigma \mathbb{C} P_{-1}^{\infty} \rightarrow$ $L_{-1}^{\infty}$ and $g: L_{-1}^{\infty} \rightarrow \Sigma^{\infty}\left(B C_{p}\right)_{+}$correspond to the maps $f_{2}$ and $g_{2}$ in diagram (1-2), respectively.

The $C_{p}$-transfer map $t_{p}$ induces multiplication by $p$ on $\pi_{0}$, and the zero map in mod $p$ homology, so $\pi_{-1}(f)$ is surjective, $f_{*}$ maps $\Sigma \beta_{-1}$ to $\alpha_{-1}$, and $g_{*}$ maps $\alpha_{k}$ to $\alpha_{k}$ for all $k \geq 0$. It remains to see that $g_{*} f_{*}$ maps $\Sigma \beta_{k}$ to $\alpha_{2 k+1}$ for $k \geq 0$. This is clear from diagram (1-3), since $g_{3} f_{3}$ agrees in positive degrees with the $\mathbb{T}$-transfer map $\Sigma^{\infty} \Sigma\left(\mathbb{C} P_{+}^{\infty}\right) \rightarrow \Sigma^{\infty}\left(B C_{p}\right)_{+}$, which has this behavior on homology.

\section{Algebraic $K$-theory of finite $G$-sets}

In this section we will compare the algebraic $K$-theory spectrum of finite $G$-sets with the $G$-fixed points of the sphere spectrum, as structured ring spectra. Before we state the result we recall some of the definitions involved.

The $K$-theory construction we use is that of Elmendorf and Mandell [8]. When the input category is a bipermutative category $\mathcal{C}$, their machine produces a symmetric spectrum $K(\mathcal{C})$, in the sense of Hovey, Shipley and Smith [11], with an action of the simplicial Barratt-Eccles operad. We will use the same notation for the geometrically realized symmetric spectrum in topological spaces, which has an action

$$
\kappa_{j}: E \Sigma_{j} \ltimes_{\Sigma_{j}} K(\mathcal{C})^{\wedge j} \rightarrow K(\mathcal{C})
$$


of the operad $E \Sigma$ consisting of the contractible $\Sigma_{j}$-free spaces $E \Sigma_{j}$. As usual, $E \Sigma_{j}$ can be defined as the nerve $N \widetilde{\Sigma}_{j}$ of the translation category $\widetilde{\Sigma}_{j}$, for $j \geq 0$. The $K$-theory construction itself is somewhat involved, but all we need to know is that the zeroth space $K\left(\widetilde{C}_{\widetilde{\Sigma}}\right)_{0}$ is the nerve $N \mathcal{C}$ of $\mathcal{C}$, so the zeroth space of $E \Sigma_{j} \ltimes \Sigma_{j} K(\mathcal{C})^{\wedge j}$ is the nerve of $\widetilde{\Sigma}_{j} \ltimes_{\Sigma_{j}} \mathcal{C}^{j}$, and the action of $E \Sigma$ on $K(\mathcal{C})_{0}$ is given by the maps $\lambda_{j}: E \Sigma_{j} \ltimes_{\Sigma_{j}} N \mathcal{C}^{\wedge j} \rightarrow N \mathcal{C}$ that are induced by the functors that take an object $\left(\sigma ; a_{1}, \ldots, a_{j}\right)$ in $\widetilde{\Sigma}_{j} \ltimes_{\Sigma_{j}} \mathcal{C}^{j}$ to the object $a_{\sigma^{-1}(1)} \otimes \cdots \otimes a_{\sigma^{-1}(j)}$ in $\mathcal{C}$ (see Elmendorf and Mandell [8, Section 8]). Here $\otimes$ denotes the product in the bipermutative structure on $\mathcal{C}$. Hence there is a commutative diagram

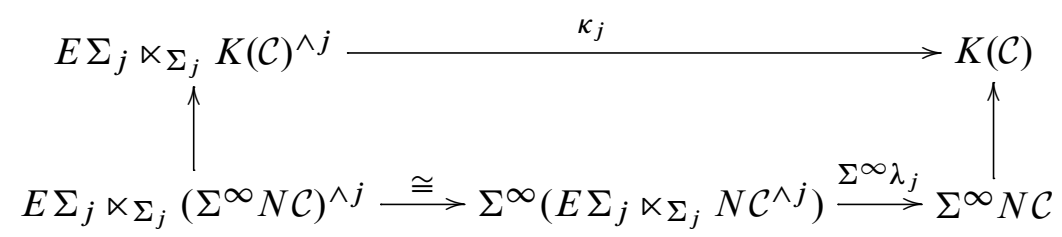

for each $j \geq 0$.

Let $G$ be a finite group, and let $\mathcal{E}^{G}$ denote the category of finite $G$-sets and $G$ equivariant bijections. This is a symmetric bimonoidal category under disjoint union and cartesian product, taking $(X, Y)$ to $X \amalg Y$ and $X \times Y$, respectively. We give $X \times Y$ the diagonal $G$-action. There is a functorially defined bipermutative category $\Phi \mathcal{E}^{G}$, and a natural equivalence $\mathcal{E}^{G} \rightarrow \Phi \mathcal{E}^{G}$ [16, VI.3.5]. It follows that there is a homotopy commutative diagram

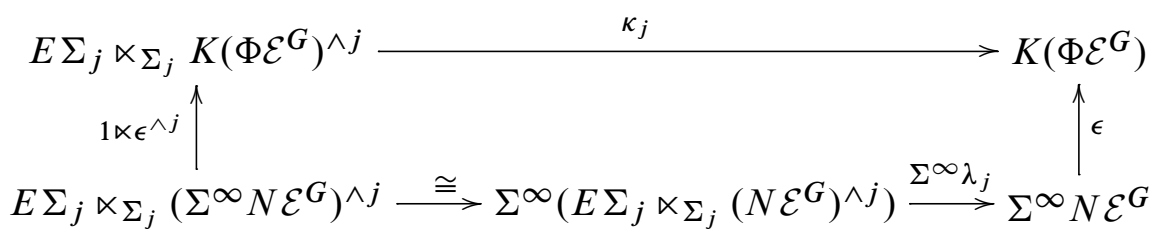

for each $j \geq 0$, where

$$
\lambda_{j}: E \Sigma_{j} \ltimes \Sigma_{j}\left(N \mathcal{E}^{G}\right)^{\wedge j} \rightarrow N \mathcal{E}^{G}
$$

is induced by the functor $\widetilde{\Sigma}_{j} \ltimes_{\Sigma_{j}}\left(\mathcal{E}^{G}\right)^{j} \rightarrow \mathcal{E}^{G}$ that takes $\left(\sigma ; X_{1}, \ldots, X_{j}\right)$ to the cartesian product

$$
X_{\sigma^{-1}(1)} \times\left(X_{\sigma^{-1}(2)} \times \cdots \times\left(X_{\sigma^{-1}(j-1)} \times X_{\sigma^{-1}(j)}\right) \cdots\right) .
$$

Let $\mathcal{U}$ be a complete $G$-universe, and let $\mathcal{L}$ denote the linear isometries operad with spaces $\mathcal{L}(j)$ consisting of linear isometries $\mathcal{U}^{j} \rightarrow \mathcal{U}$, where $\mathcal{U}^{j}$ denotes the direct sum of $j$ copies of $\mathcal{U}$. There is an action of $G$ on each $\mathcal{L}(j)$ given by conjugation, and this gives $\mathcal{L}$ the structure of an $E_{\infty} G$-operad in the sense of Lewis, May and 
Steinberger [14, VII.1.1]. The $E_{\infty}$ ring structure on the $G$-equivariant sphere spectrum $\mathbb{S}_{G}=\Sigma_{G}^{\infty} S^{0}$ is given by an action

$$
\zeta_{j}: \mathcal{L}(j) \ltimes_{\Sigma_{j}} \mathbb{S}_{G}^{\wedge j} \rightarrow \mathbb{S}_{G}
$$

of this operad (where, for once, $\ltimes$ denotes the twisted half-smash product in Lewis-May spectra). It is compatible with a corresponding action

$$
\omega_{j}: \mathcal{L}(j) \ltimes_{\Sigma_{j}} Q_{G}\left(S^{0}\right)^{\wedge j} \rightarrow Q_{G}\left(S^{0}\right)
$$

on the underlying infinite loop space $Q_{G}\left(S^{0}\right)=\Omega^{\infty} \mathbb{S}_{G}=\operatorname{colim}_{V \subset \mathcal{U}} \Omega^{V} S^{V}$, in the sense that the following diagram commutes.

$$
\begin{aligned}
& \mathcal{L}(j) \ltimes_{\Sigma_{j}}\left(\Sigma^{\infty} Q_{G}\left(S^{0}\right)\right)^{\wedge j} \stackrel{\cong}{\longrightarrow} \Sigma^{\infty}\left(\mathcal{L}(j) \ltimes \Sigma_{j} Q_{G}\left(S^{0}\right)^{\wedge j}\right) \stackrel{\Sigma^{\infty} \omega_{j}}{\longrightarrow} \Sigma^{\infty} Q_{G}\left(S^{0}\right) \\
& 1 \ltimes \epsilon^{\wedge j} \downarrow \\
& \mathcal{L}(j) \ltimes \Sigma_{j} \mathbb{S}_{G}^{\wedge j}
\end{aligned}
$$

Here $\omega_{j}$ sends an element in $\mathcal{L}(j) \ltimes_{\Sigma_{j}} Q_{G}\left(S^{0}\right)^{\wedge j}$ represented by $\left(f ; g_{1}, \ldots, g_{j}\right)$, where $f: \mathcal{U}^{j} \rightarrow \mathcal{U}$ and $g_{i}: S^{V_{i}} \rightarrow S^{V_{i}}$, to the element represented by the composite of the following maps.

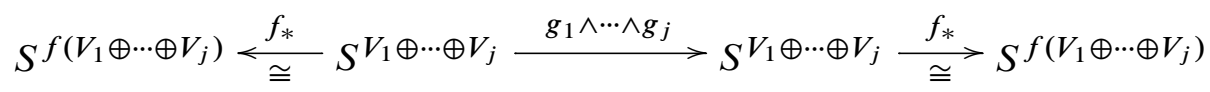

By taking $G$-fixed points we get the nonequivariant $E_{\infty}$ ring spectrum $\mathbb{S}^{G}=\left(\mathbb{S}_{G}\right)^{G}$ with an action

$$
\xi_{j}: \mathcal{L}^{G}(j) \ltimes \Sigma_{j}\left(\mathbb{S}^{G}\right)^{\wedge j} \rightarrow \mathbb{S}^{G}
$$

of the nonequivariant $E_{\infty}$ operad $\mathcal{L}^{G}$ of $G$-equivariant isometries. The corresponding infinite loop space $\Omega^{\infty}\left(\mathbb{S}^{G}\right)$ is the space $Q_{G}\left(S^{0}\right)^{G}=\operatorname{colim}_{V \subset \mathcal{U}}\left(\Omega^{V} S^{V}\right)^{G}$, with the inherited $\mathcal{L}^{G}$-action

$$
\eta_{j}: \mathcal{L}^{G}(j) \ltimes_{\Sigma_{j}}\left(Q_{G}\left(S^{0}\right)^{G}\right)^{\wedge j} \rightarrow Q_{G}\left(S^{0}\right)^{G} .
$$

Next we recall the definition of the Dyer-Lashof operations $Q^{i}$. Let $C_{*}(-)$ denote the cellular chains functor, from either $\mathrm{CW}$ complexes or $\mathrm{CW}$ spectra to chain complexes. Let $E$ be a spectrum with an action of an $E_{\infty}$ operad $\mathcal{O}$, and let $W_{*}$ be the standard free $C_{p}$-resolution of $\mathbb{F}_{p}$ with basis elements $e_{i}$ in degree $i$. There is a chain map $W_{*} \rightarrow C_{*}(\mathcal{O}(p))$ lifting the identity on $\mathbb{F}_{p}$, unique up to homotopy, and we also denote the image of $e_{i}$ under this map by $e_{i}$. Let $x \in H_{q}(E)$ be represented by a cycle 
$z \in C_{q}(E)$. Now consider the image of the cycle $e_{i} \otimes z^{\otimes p}$ under the map

$$
C_{*}(\mathcal{O}(p)) \otimes_{\Sigma_{p}} C_{*}(E)^{\otimes p} \stackrel{\cong}{\longrightarrow} C_{*}\left(\mathcal{O}(p) \ltimes \Sigma_{p} E^{\wedge p}\right) \stackrel{\xi_{p *}}{\longrightarrow} C_{*}(E),
$$

and denote its image in homology by $Q_{i}(x)$. Here $\xi_{p}$ is the $E_{\infty}$ structure map. Then for $p=2$ define $Q^{i}(x)=0$ when $i<q$, and

$$
Q^{i}(x)=Q_{i-q}(x)
$$

when $i \geq q$. For $p>2$ define $Q^{i}(x)=0$ when $2 i<q$, and

$$
Q^{i}(x)=(-1)^{i} v(q) \cdot Q_{(2 i-q)(p-1)}(x)
$$

when $2 i \geq q$, where $v(q)=(-1)^{q(q-1)(p-1) / 4}\left(\left(\frac{1}{2}(p-1)\right) !\right)^{q}$. See Bruner, May, McClure and Steinberger [2, Chapter III] for more details.

The spectra $\mathbb{S}^{G}$ and $K\left(\Phi \mathcal{E}^{G}\right)$ should be equivalent as $E_{\infty}$ ring spectra, but we will only need the following weaker result.

Lemma 2.1 There is an equivalence $\mathbb{S}^{G} \simeq K\left(\Phi \mathcal{E}^{G}\right)$ of spectra such that the induced isomorphism $H_{*}\left(\mathbb{S}^{G}\right) \cong H_{*}\left(K\left(\Phi \mathcal{E}^{G}\right)\right)$ commutes with the Dyer-Lashof operations.

Proof Our first goal is to construct a commutative diagram:

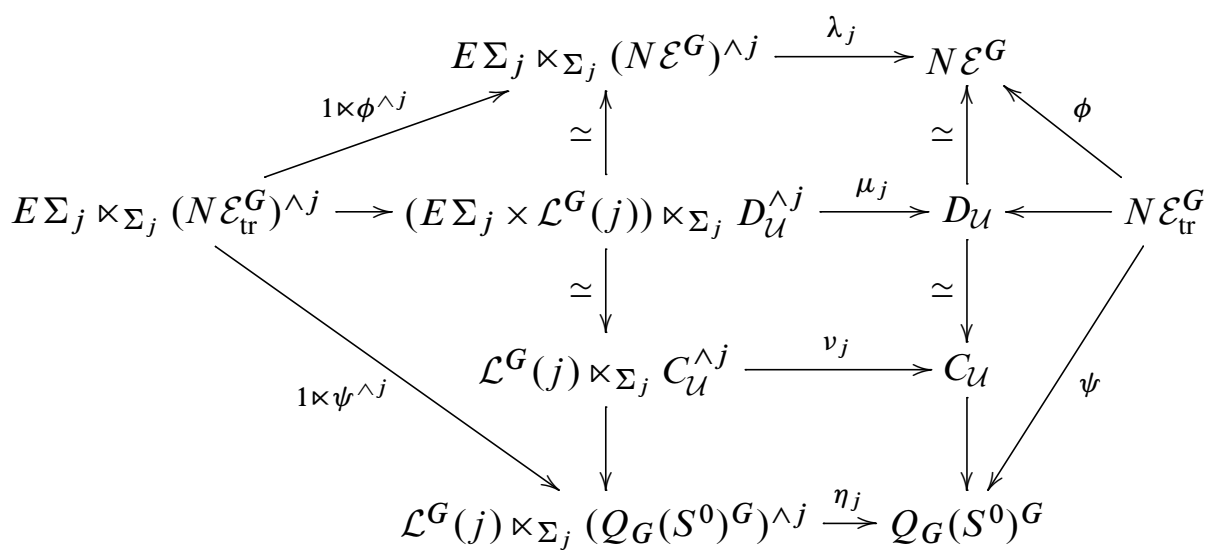

We start by describing the space $C_{\mathcal{U}}$. Let $V$ be an indexing space in $\mathcal{U}$. For each finite $G$-set $X$, consider the space $E_{V}(X)$ of $X$-tuples of distance-reducing embeddings of $V$ in $V$, closed under the action of $G$. More precisely, this is the space of $G-$ equivariant maps $\bigsqcup_{X} V \rightarrow V$ such that the restriction to each summand $g: V \rightarrow V$ is an embedding that satisfies $|g(v)-g(w)| \leq|v-w|$ for all $v, w \in V$. Let $K_{V}(X)$ 
be the space of paths $[0,1] \rightarrow E_{V}(X)$ such that the embeddings at the endpoint 0 are identities, and the embeddings at 1 have disjoint images. Now let

$$
K_{\mathcal{U}}(X)=\operatorname{colim}_{V \subset \mathcal{U}} K_{V}(X) .
$$

These are $G$-equivariant versions of the spaces in the Steiner operad [22]. The group $\operatorname{Aut}^{G}(X)$ acts freely on $K_{\mathcal{U}}(X)$ by permuting the embeddings, and the space $C_{\mathcal{U}}$ is to be the disjoint union

$$
C_{\mathcal{U}}=\coprod_{[X]} K_{\mathcal{U}}(X) / \operatorname{Aut}^{G}(X)
$$

where $X$ ranges over all isomorphism classes of finite $G$-sets.

The action of the operad $\mathcal{L}^{G}$ on $C_{\mathcal{U}}$ is defined as follows. Let $f: \mathcal{U}^{j} \rightarrow \mathcal{U}$ be a $G$-linear isometry, and let $\left[g_{i}\right], 1 \leq i \leq j$, be elements in $C_{\mathcal{U}}$, represented by paths of $X_{i}$-tuples of embeddings $g_{i} \in K_{\mathcal{U}}\left(X_{i}\right)$. Denote the component paths of embeddings that constitute $g_{i}$ by $g_{i, x_{i}}$, where $x_{i} \in X_{i}$. The resulting element $v_{j}\left(f ;\left[g_{1}\right], \ldots,\left[g_{j}\right]\right)$ in $C_{\mathcal{U}}$ is represented by an element in $K_{\mathcal{U}}\left(X_{1} \times \cdots \times X_{j}\right)$, which on the summand indexed by $\left(x_{1}, \ldots, x_{j}\right)$ is given by $f \circ\left(g_{1, x_{1}} \times \cdots \times g_{j, x_{j}}\right) \circ f^{-1}$.

There is a map $C_{\mathcal{U}} \rightarrow Q_{G}\left(S^{0}\right)^{G}$, given by evaluating a Steiner path in $E_{V}(X)$ at 1 to get a $G$-equivariant embedding $e: \bigsqcup_{X} V \rightarrow V$, and then applying a folded PontryaginThom construction to obtain a $G$-equivariant map $q: S^{V} \rightarrow S^{V}$, which is a point in $Q_{G}\left(S^{0}\right)^{G}$. Given the distance-reducing embedding $e$, let $S^{V} \rightarrow \bigvee_{X} S^{V}$ be the $G$-equivariant map that is given by $e^{-1}$ on the image of $e$ in $V \subset S^{V}$ and maps the remainder of $S^{V}$ to the base point of $\bigvee_{X} S^{V} \supset \bigsqcup_{X} V$. Let $\bigvee_{X} S^{V} \rightarrow S^{V}$ be the fold map that is the identity on each summand. The folded Pontryagin-Thom construction $q$ is the composite of these two $G$-maps. If we permute the embeddings indexed by $X$ we get the same element in $Q_{G}\left(S^{0}\right)^{G}$, so our map is well-defined. A comparison of definitions shows that this construction is compatible with the $\mathcal{L}^{G}$-actions on $C_{\mathcal{U}}$ and $Q_{G}\left(S^{0}\right)^{G}$, so the lower square in (2-3) commutes.

Let

$$
D_{\mathcal{U}}=\coprod_{[X]}\left(E \operatorname{Aut}^{G}(X) \times K_{\mathcal{U}}(X)\right) / \operatorname{Aut}^{G}(X),
$$

where $\operatorname{Aut}^{G}(X)$ acts diagonally on the product. The nerve $N \mathcal{E}^{G}$ splits as a sum of components

$$
N \mathcal{E}^{G} \simeq \coprod_{[X]} B \operatorname{Aut}^{G}(X)
$$

where the disjoint union is over the isomorphism classes of $G$-sets $X$. Projection on the first factor in $D_{\mathcal{U}}$ followed by this homotopy equivalence gives the map 
$D_{\mathcal{U}} \rightarrow N \mathcal{E}^{G}$ in (2-3), while the map $D_{\mathcal{U}} \rightarrow C_{\mathcal{U}}$ is the projection on the second factor. There is an induced action of the product operad $E \Sigma \times \mathcal{L}^{G}$ on $D_{\mathcal{U}}$, defined as follows. Let $(e, f) \in E \Sigma_{j} \times \mathcal{L}^{G}(j)$ and let $\left(e_{i}, f_{i}\right) \in E \operatorname{Aut}^{G}(X) \times C_{\mathcal{U}}(X)$ represent elements in $D_{\mathcal{U}}$, for $1 \leq i \leq j$. The image under $\mu_{j}$ is the element represented by $\left(\lambda_{j}\left(e ; e_{1}, \ldots, e_{j}\right), v_{j}\left(f ; f_{1}, \ldots, f_{j}\right)\right)$. This makes the upper and middle squares in (2-3) commute.

Let

$$
N \mathcal{E}_{\text {tr }}^{G}=\left(\coprod_{(H)}\left(E \operatorname{Aut}^{G}(G / H) \times K_{\mathcal{U}}(G / H)\right) / \operatorname{Aut}^{G}(G / H)\right)_{+},
$$

where the coproduct is taken over the conjugacy classes of subgroups $H$ of $G$. The map $N \mathcal{E}_{\text {tr }}^{G} \rightarrow D_{\mathcal{U}}$ in (2-3) is the inclusion of the components indexed by the isomorphism classes of transitive $G$-sets.

The maps $\phi$ and $\psi$ are defined by commutativity of the right hand triangles in the diagram. We claim that the adjoints

$$
\begin{aligned}
& \Sigma^{\infty} N \mathcal{E}_{\mathrm{tr}}^{G} \rightarrow K\left(\mathcal{E}^{G}\right) \simeq K\left(\Phi \mathcal{E}^{G}\right) \\
& \Sigma^{\infty} N \mathcal{E}_{\mathrm{tr}}^{G} \rightarrow \mathbb{S}^{G}
\end{aligned}
$$

of the maps $\phi$ and $\psi$, respectively, are both equivalences. Here $K\left(\mathcal{E}^{G}\right)$ is the additive $K$-theory spectrum of $\mathcal{E}^{G}$, with zeroth space $K\left(\mathcal{E}^{G}\right)_{0}=N \mathcal{E}^{G}$, which only depends on the additive symmetric monoidal structure of $\mathcal{E}^{G}$. There is an equivalence

$$
\Sigma^{\infty} N \mathcal{E}_{\mathrm{tr}}^{G} \simeq \bigvee_{(H)} \Sigma^{\infty} B W_{G} H_{+},
$$

where $W_{G} H=N_{G} H / H \cong \operatorname{Aut}^{G}(G / H)$ is the Weyl group of $H$ and the wedge sum is over the conjugacy classes of subgroups of $G$. By Waldhausen's additivity theorem $[23,1.3 .2]$ applied to a suitable filtration of $\mathcal{E}^{G}$ according to stabilizer types, there is a splitting

$$
K\left(\mathcal{E}^{G}\right) \simeq \bigvee_{(H)} K\left(\mathcal{E}\left(W_{G} H\right)\right)
$$

where $\mathcal{E}\left(W_{G} H\right)$ is the category of finite free $W_{G} H$-sets and equivariant bijections. The map (2-5) is equivalent under these identifications to the wedge sum of the maps

$$
\Sigma^{\infty} B W_{G} H_{+} \rightarrow K\left(\mathcal{E}\left(W_{G} H\right)\right)
$$

that are left adjoint to the inclusions $B W_{G} H_{+} \rightarrow N \mathcal{E}\left(W_{G} H\right)=K\left(\mathcal{E}\left(W_{G} H\right)\right)_{0}$.

The Barratt-Priddy-Quillen-Segal theorem [20, 3.6] says that each of the maps (2-7) is an equivalence, hence (2-5) is an equivalence. The map (2-6) is an equivalence by 
the Segal-tom Dieck splitting [14, V.11.2]. The composition of these two equivalences is the equivalence $\mathbb{S}^{G} \simeq K\left(\Phi \mathcal{E}^{G}\right)$ referred to in the statement of the lemma.

We apply the suspension spectrum functor $\Sigma^{\infty}$ to the diagram (2-3), combine it with diagram (2-1) and the $G$-fixed part of (2-2), take homology, and end up with the following commutative diagram.

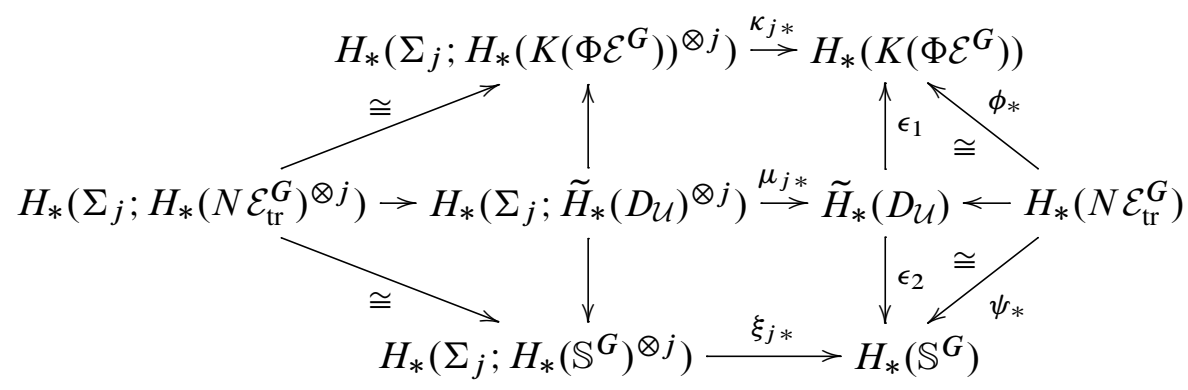

We need the fact that $\epsilon_{1}$ and $\epsilon_{2}$ have the same kernel. In fact, all summands in $\widetilde{H}_{*}\left(D_{\mathcal{U}}\right)$ indexed by $G$-sets with more than one orbit map to zero under both $\epsilon_{1}$ and $\epsilon_{2}$. This follows from the fact that Pontryagin products and additive Dyer-Lashof operations vanish after stabilization. More precisely, a decomposition of a $G$-set $X=$ $\coprod_{i=1}^{k} n_{i}\left(G / H_{i}\right)$, where the $H_{i}$ lie in distinct conjugacy classes, induces a factorization

$$
\operatorname{BAut}^{G}(X) \cong \prod_{i=1}^{k} B\left(\Sigma_{n_{i}} \prec W_{G} H_{i}\right) .
$$

The homology group $H_{*}\left(B\left(\Sigma_{n_{i}} 2 W_{G} H_{i}\right)\right) \subset H_{*}\left(N \mathcal{E}^{G}\right)$ is generated by $H_{*}\left(B W_{G} H_{i}\right)$ under iterated Pontryagin products and Dyer-Lashof operations (see Cohen, Lada and May [3, I.4.1]), which all map to zero under $\epsilon_{1}$ and $\epsilon_{2}$ unless $k=1$ and $n_{1}=1$.

Let $x \in H_{*}\left(K\left(\Phi \mathcal{E}^{G}\right)\right)$, and let $y \in H_{*}\left(\mathbb{S}^{G}\right)$ be the element corresponding to $x$ under $\psi_{*} \circ \phi_{*}^{-1}$, via an element $z \in H_{*}\left(N \mathcal{E}_{\text {tr }}^{G}\right)$. We need to show that the image $Q_{i}(x)$ of $e_{i} \otimes x^{\otimes p}$ under the top map corresponds, via the isomorphism, to the image $Q_{i}(y)$ of $e_{i} \otimes y^{\otimes p}$ under the bottom map. The element $e_{i} \otimes z^{\otimes p} \in H_{*}\left(\Sigma_{p} ; H_{*}\left(N \mathcal{E}_{\text {tr }}^{G}\right)^{\otimes p}\right)$ maps to an element $Q_{i}(z) \in \tilde{H}_{*}\left(D_{\mathcal{U}}\right)$, which further maps to $Q_{i}(x)$ and $Q_{i}(y)$ under $\epsilon_{1}$ and $\epsilon_{2}$, respectively. Let $w \in H_{*}\left(N \mathcal{E}_{\text {tr }}^{G}\right)$ map to $Q_{i}(x)$ under $\phi_{*}$. Since the maps $\epsilon_{1}$ and $\epsilon_{2}$ have the same kernel, the elements $Q_{i}(z)$ and $w$ have the same image in $H_{*}\left(\mathbb{S}^{G}\right)$, which implies the result.

Remark 2.2 The additive equivalence $\mathbb{S}^{G} \simeq K\left(\mathcal{E}^{G}\right) \simeq K\left(\Phi \mathcal{E}^{G}\right)$ of spectra can be realized as the $G$-fixed part of a $G$-equivalence $\mathbb{S}_{G} \simeq K_{G}(\mathcal{E})$ of $G$-spectra, for example using Shimakawa's construction [21] of $G$-equivariant $K$-theory spectra. Presumably this is a $G$-equivalence of $E_{\infty}$ ring $G$-spectra. 


\section{Proof of the main theorem}

Recall the $E_{\infty}$ structure maps $\lambda_{j}: E \Sigma_{j} \ltimes \Sigma_{j}\left(N \mathcal{E}^{G}\right)^{\wedge j} \rightarrow N \mathcal{E}^{G}$. We have inclusions $B \operatorname{Aut}^{G}(G) \rightarrow N \mathcal{E}^{G}$ and $\delta: B$ Aut $^{G}\left(G^{j}\right) \rightarrow N \mathcal{E}^{G}$, corresponding to the summands indexed by $X=G$ and $X=G^{j}=G \times \cdots \times G$, respectively, in the decomposition (2-4) of $N \mathcal{E}^{G}$. Restricting $\lambda_{j}$ to these summands, we have a commutative diagram

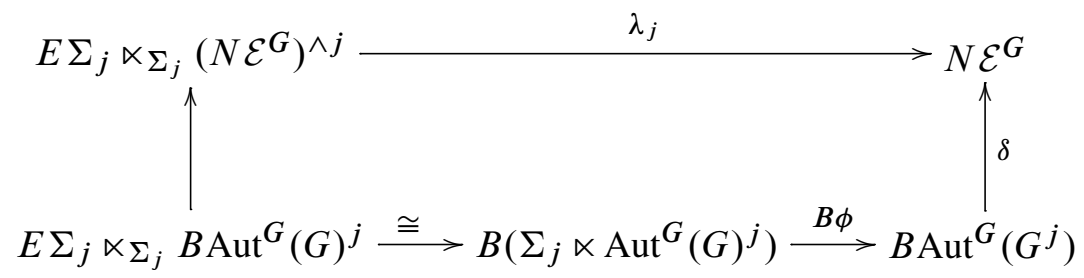

where the homomorphism $\phi$ sends an element $\left(\sigma ; f_{1}, \ldots, f_{j}\right)$ in $\Sigma_{j} \ltimes \operatorname{Aut}^{G}(G)^{j}$ to the $G$-automorphism $f_{\sigma^{-1}(1)} \times \cdots \times f_{\sigma^{-1}(j)}$ of $G^{j}$.

We write $\Sigma_{j}<\operatorname{Aut}^{G}(G) \cong \Sigma_{j} \prec G$ for the wreath product $\Sigma_{j} \ltimes \operatorname{Aut}^{G}(G)^{j}$. The free $G$-set $G^{j}$ splits into $k=|G|^{j-1}$ orbits, and we fix a $G$-equivariant bijection $G^{j} \cong \bigsqcup_{k} G$. This induces an isomorphism $\operatorname{Aut}^{G}\left(G^{j}\right) \cong \operatorname{Aut}^{G}\left(\coprod_{k} G\right)$, and we also have $\operatorname{Aut}^{G}\left(\coprod_{k} G\right) \cong \Sigma_{k} \succ G$. Thus we get a commutative diagram

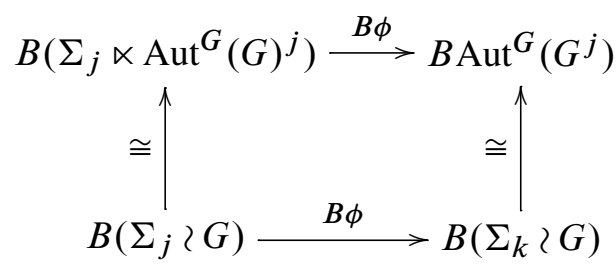

where we also write $\phi$ for the induced homomorphism $\Sigma_{j} \prec G \rightarrow \Sigma_{k} \prec G$.

Now we specialize to the case $p=2$. First we study the Dyer-Lashof operation $Q^{2}: H_{1}\left(\mathbb{S}^{C_{2}}\right) \rightarrow H_{3}\left(\mathbb{S}^{C_{2}}\right)$.

Lemma 3.1 The operation $Q^{2}$ in $H_{*}\left(\mathbb{S}^{C_{2}}\right)$ satisfies $Q^{2}\left(\alpha_{1}\right)=\alpha_{3}$.

Proof Let $C=C_{2}$. By Lemma 2.1, we may instead compute $Q^{2}$ in $H_{*}\left(K\left(\Phi \mathcal{E}^{C}\right)\right)$. We let $j=2$, combine diagrams (2-1), (3-1) and (3-2), apply homology, and end up 
with the upper half of the diagram:

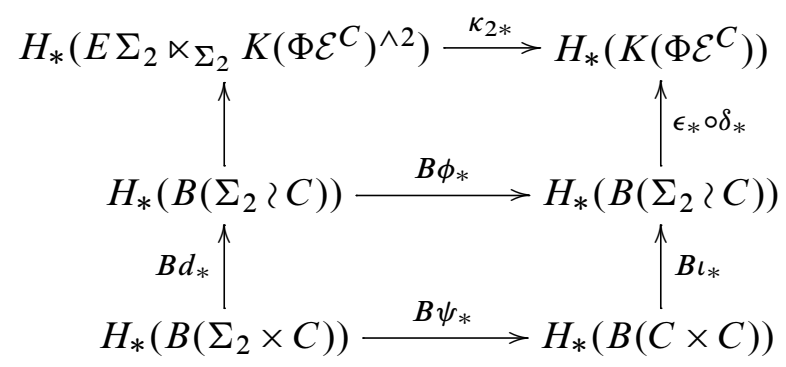

The vertical homomorphisms in the lower square are induced by the homomorphism $d=1 \times \Delta$ that sends $(\sigma, x)$ to $(\sigma ; x, x)$, and the inclusion $\iota$ of the subgroup $C \times C=C^{2}$ in $\Sigma_{2} \prec C=\Sigma_{2} \ltimes C^{2}$. The homomorphism $\psi$ is the restriction of $\phi$ to $\Sigma_{2} \times C$. It is easily checked that $\psi$ takes values in the subgroup $C \times C$ (since $p=2$ ) and is given by $\psi(\sigma, x)=(x, \sigma x)$, using the description of $\phi$ given after diagram (3-1). We have $B \psi_{*}\left(e_{i} \otimes 1\right)=1 \otimes \alpha_{i}$ and

$$
B \psi_{*}\left(1 \otimes \alpha_{j}\right)=\Delta_{*}\left(\alpha_{j}\right)=\sum_{s+t=j} \alpha_{s} \otimes \alpha_{t},
$$

which combine to give

$$
B \psi_{*}\left(e_{i} \otimes \alpha_{j}\right)=\sum_{s+t=j} \alpha_{s} \otimes\left(\alpha_{i} * \alpha_{t}\right),
$$

where $*$ denotes the Pontryagin product in $H_{*}(B C)$ induced by the topological group multiplication $B C \times B C \rightarrow B C$. We recall that $\alpha_{i} * \alpha_{t}=\left(\begin{array}{c}i+t \\ i\end{array}\right) \alpha_{i+t}$.

By May's paper $[15,9.1]$ the map $B d_{*}$ is given by

$$
B d_{*}\left(e_{i} \otimes \alpha_{j}\right)=\sum_{k} e_{i+2 k-j} \otimes \operatorname{Sq}_{*}^{k}\left(\alpha_{j}\right) \otimes \operatorname{Sq}_{*}^{k}\left(\alpha_{j}\right) .
$$

Recall that $\mathrm{Sq}_{*}^{k}\left(\alpha_{j}\right)=\left(\begin{array}{c}j-k \\ k\end{array}\right) \alpha_{j-k}$, where $\mathrm{Sq}_{*}^{k}$ denotes the dual of the Steenrod operation $\mathrm{Sq}^{k}$. In particular $B d_{*}\left(e_{1} \otimes \alpha_{2}\right)=e_{1} \otimes \alpha_{1} \otimes \alpha_{1}$, which further maps to $Q_{1}\left(\alpha_{1}\right)$ in the upper right hand corner of (3-3). But now $Q_{1}\left(\alpha_{1}\right)$ is also the image of $e_{1} \otimes \alpha_{2}$ under $\epsilon_{*} \circ \delta_{*} \circ B \iota_{*} \circ B \psi_{*}$. Using the description of $B \psi_{*}$ above, we see that $Q_{1}\left(\alpha_{1}\right)$ equals

$$
\sum_{s+t=2} \epsilon_{*} \delta_{*}\left(1 \otimes \alpha_{S} \otimes\left(\alpha_{1} * \alpha_{t}\right)\right)
$$

The map $\epsilon_{*}$ vanishes on decomposables with respect to the product in $H_{*}\left(N \mathcal{E}^{C}\right)$ which is induced by the additive symmetric monoidal structure on $\mathcal{E}^{C}$. The element 
$\delta_{*}\left(1 \otimes \alpha_{s} \otimes\left(\alpha_{1} * \alpha_{t}\right)\right)$ is the image of

$$
\alpha_{s} \otimes\left(\alpha_{1} * \alpha_{t}\right) \in H_{*}\left(\operatorname{BAut}^{C}(C) \times \text { BAut }^{C}(C)\right)
$$

in $H_{*}\left(B\right.$ Aut $\left.{ }^{C}(C \amalg C)\right) \subset H_{*}\left(N \mathcal{E}^{C}\right)$ under the map induced by disjoint union, thus the only nonzero term in (3-4) is the one with $s=0$ and $t=2$, and $Q^{2}\left(\alpha_{1}\right)=$ $Q_{1}\left(\alpha_{1}\right)=\epsilon_{*}\left(1 \otimes \alpha_{0} \otimes\left(\alpha_{1} * \alpha_{2}\right)\right)=\alpha_{3}$.

Proof of Theorem 0.2 We now turn to the operations in

$$
H_{*}\left(\mathrm{TC}^{(1)}(\star ; 2)\right)=\mathbb{F}_{2} \oplus H_{*}\left(\mathbb{R} P_{-1}^{\infty}\right) .
$$

The general formula for the $Q^{i}$ will follow from Lemma 3.1 and the Nishida relations, which say in particular (see Bruner et al [2, III.1.1]) that

$$
\mathrm{Sq}_{*}^{i+j+1} Q^{i}\left(\alpha_{j}\right)=\sum_{k}\left(\begin{array}{c}
2^{N}-j-1 \\
2^{N}-i-2 j-2+2 k
\end{array}\right) Q^{k-j-1} \mathrm{Sq}_{*}^{k}\left(\alpha_{j}\right),
$$

where $N$ is sufficiently large. When $k \geq j+2$ the element $\operatorname{Sq}_{*}^{k}\left(\alpha_{j}\right)$ is zero for degree reasons, and when $k \leq j$ the fact that $Q^{k-j-1}$ vanishes on classes in degree higher than $k-j-1$ implies that $Q^{k-j-1} \mathrm{Sq}_{*}^{k}\left(\alpha_{j}\right)=0$. Hence the sum in (3-5) simplifies to the single term

$$
\mathrm{Sq}_{*}^{i+j+1} Q^{i}\left(\alpha_{j}\right)=\left(\begin{array}{c}
2^{N}-j-1 \\
2^{N}-i
\end{array}\right) Q^{0} \mathrm{Sq}_{*}^{j+1}\left(\alpha_{j}\right)
$$

for $k=j+1$, where $N$ is large.

Bob Bruner has observed that

$$
\left(\begin{array}{c}
2^{N}-j-1 \\
2^{N}-i
\end{array}\right) \equiv\left(\begin{array}{c}
2^{N}+i-1 \\
2^{N}+j
\end{array}\right)
$$

$\bmod 2$, for large $N$. Here is a quick proof. Let $x_{k}$ denote the $k$-th bit in the binary expansion of a natural number $x$. Then

$$
\left(\begin{array}{c}
2^{N}-j-1 \\
2^{N}-i
\end{array}\right) \equiv 1
$$

if and only if $\left(2^{N}-i\right)_{k}=1$ implies $\left(2^{N}-j-1\right)_{k}=1$ for all $k$, and

$$
\left(\begin{array}{c}
2^{N}+i-1 \\
2^{N}+j
\end{array}\right) \equiv 1
$$

if and only if $\left(2^{N}+j\right)_{k}=1$ implies $\left(2^{N}+i-1\right)_{k}=1$ for all $k$. But for $N$ large compared to $i, j$ and $k$ the bit $\left(2^{N}-i\right)_{k}$ is complementary to $\left(2^{N}+i-1\right)_{k}$, and 
$\left(2^{N}-j-1\right)_{k}$ is complementary to $\left(2^{N}+j\right)_{k}$, so

$$
\left(\begin{array}{c}
2^{N}-j-1 \\
2^{N}-i
\end{array}\right) \equiv 1
$$

if and only if

$$
\left(\begin{array}{c}
2^{N}+i-1 \\
2^{N}+j
\end{array}\right) \equiv 1
$$

The operations $\mathrm{Sq}_{*}^{k}$ in $H_{*}\left(\mathbb{R} P_{-1}^{\infty}\right)$ are given by the formula

$$
\mathrm{Sq}_{*}^{k}\left(\alpha_{j}\right)=\left(\begin{array}{c}
j-k \\
k
\end{array}\right) \alpha_{j-k}
$$

This follows by the corresponding formula for $\mathbb{R} P^{\infty}$ and James periodicity. More precisely, a theorem of James [12] says that given $m \leq n$, there is a positive integer $M$, depending only on $n-m$, such that $\mathbb{R} P_{m+\ell}^{n+\ell} \simeq \Sigma^{\ell} \mathbb{R} P_{m}^{n}$ when $\ell$ is a positive multiple of $2^{M}$. The space $\mathbb{R} P_{m}^{n}$ is the stunted projective space $\mathbb{R} P^{n} / \mathbb{R} P^{m-1}$. If we now define the spectrum $\mathbb{R} P_{-1}^{n}$ to be $\Sigma^{-\ell} \Sigma^{\infty} \mathbb{R} P_{\ell-1}^{n+\ell}$ for such $\ell$ (depending on $n$ ), we have that $\mathbb{R} P_{-1}^{\infty}=\operatorname{colim}_{n} \mathbb{R} P_{-1}^{n}$. The Steenrod operations in $H_{*}\left(\mathbb{R} P_{-1}^{\infty}\right)$ can now be calculated from the operations in $H_{*}\left(\mathbb{R} P_{\ell-1}^{n+\ell}\right)$, and the stated formula follows by noting that the relevant binomial coefficients are $2^{M}$-periodic in the numerator.

In particular $\mathrm{Sq}_{*}^{j+1}\left(\alpha_{j}\right)=\alpha_{-1}$ for all $j \geq-1$, and we have

$$
\mathrm{Sq}_{*}^{i+j+1} Q^{i}\left(\alpha_{j}\right)=\left(\begin{array}{c}
2^{N}+i-1 \\
2^{N}+j
\end{array}\right) Q^{0}\left(\alpha_{-1}\right) .
$$

If $Q^{0}\left(\alpha_{-1}\right)$ were zero, it would follow that $Q^{i}\left(\alpha_{j}\right)=0$ for all $i$ and $j$, since $\mathrm{Sq}_{*}^{i+j+1}$ is an isomorphism to dimension -1 . But this contradicts Lemma 3.1. Hence $Q^{0}\left(\alpha_{-1}\right)=\alpha_{-1}$, and the formula stated in the theorem follows.

\section{References}

[1] M Bökstedt, W C Hsiang, I Madsen, The cyclotomic trace and algebraic $K$-theory of spaces, Invent. Math. 111 (1993) 465-539 MR1202133

[2] R R Bruner, JP May, J E McClure, M Steinberger, $H_{\infty}$ ring spectra and their applications, Lecture Notes in Math. 1176, Springer, Berlin (1986) MR836132

[3] F R Cohen, T J Lada, J P May, The homology of iterated loop spaces, Lecture Notes in Math. 533, Springer, Berlin (1976) MR0436146

[4] P Deligne, Le groupe fondamental de la droite projective moins trois points, from: "Galois groups over Q (Berkeley, CA, 1987)", (Y Ihara, K Ribet, J-P Serre, editors), Math. Sci. Res. Inst. Publ. 16, Springer, New York (1989) 79-297 MR1012168 
[5] B I Dundas, Relative $K$-theory and topological cyclic homology, Acta Math. 179 (1997) 223-242 MR1607556

[6] B I Dundas, P A Østvær, A bivariant Chern character, in preparation

[7] A D Elmendorf, I Kriz, M A Mandell, J P May, Rings, modules, and algebras in stable homotopy theory, Math. Surveys and Monogr. 47, Amer. Math. Soc. (1997) MR1417719 With an appendix by M Cole

[8] A D Elmendorf, M A Mandell, Rings, modules, and algebras in infinite loop space theory, Adv. Math. 205 (2006) 163-228 MR2254311

[9] T Geisser, L Hesselholt, Topological cyclic homology of schemes, from: "Algebraic $K$-theory (Seattle, WA, 1997)”, (W Raskind, C Weibel, editors), Proc. Sympos. Pure Math. 67, Amer. Math. Soc. (1999) 41-87 MR1743237

[10] L Hesselholt, I Madsen, On the $K$-theory of finite algebras over Witt vectors of perfect fields, Topology 36 (1997) 29-101 MR1410465

[11] M Hovey, B Shipley, J Smith, Symmetric spectra, J. Amer. Math. Soc. 13 (2000) 149-208 MR1695653

[12] I M James, Spaces associated with Stiefel manifolds, Proc. London Math. Soc. (3) 9 (1959) 115-140 MR0102810

[13] K-H Knapp, Some applications of $K$-theory to framed bordism: $e$-invariant and transfer, Habilitationsschrift, Universität Bonn (1979)

[14] L G Lewis, Jr, J P May, M Steinberger, J E McClure, Equivariant stable homotopy theory, Lecture Notes in Math. 1213, Springer, Berlin (1986) MR866482 With contributions by $\mathrm{J}$ E McClure

[15] J P May, A general algebraic approach to Steenrod operations, from: "The Steenrod Algebra and its Applications (Proc. Conf. to Celebrate N. E. Steenrod's Sixtieth Birthday, Battelle Memorial Inst., Columbus, Ohio, 1970)", Lecture Notes in Math. 168, Springer, Berlin (1970) 153-231 MR0281196

[16] J P May, $E_{\infty}$ ring spaces and $E_{\infty}$ ring spectra, Lecture Notes in Math. 577, Springer, Berlin (1977) MR0494077 With contributions by F Quinn, N Ray, and J Tornehave

[17] J Morava, A theory of base motives arXiv:0908.3124

[18] J Rognes, Two-primary algebraic K-theory of pointed spaces, Topology 41 (2002) 873-926 MR1923990

[19] J Rognes, The smooth Whitehead spectrum of a point at odd regular primes, Geom. Topol. 7 (2003) 155-184 MR1988283

[20] G Segal, Categories and cohomology theories, Topology 13 (1974) 293-312 MR0353298

[21] K Shimakawa, Infinite loop G-spaces associated to monoidal G-graded categories, Publ. Res. Inst. Math. Sci. 25 (1989) 239-262 MR1003787 
[22] R Steiner, A canonical operad pair, Math. Proc. Cambridge Philos. Soc. 86 (1979) 443-449 MR542690

[23] F Waldhausen, Algebraic K-theory of topological spaces. I, from: "Algebraic and geometric topology (Proc. Sympos. Pure Math., Stanford Univ., 1976), Part 1”, (R J Milgram, editor), Proc. Sympos. Pure Math. XXXII, Amer. Math. Soc. (1978) 35-60 MR520492

[24] B Williams, Bivariant Riemann Roch theorems, from: "Geometry and topology: Aarhus (1998)”, (K Grove, I H Madsen, E K Pedersen, editors), Contemp. Math. 258, Amer. Math. Soc. (2000) 377-393 MR1778119

Department of Mathematics, University of Oslo

Oslo, Norway

hakonsb@math.uio.no, rognes@math.uio.no

Proposed: Ralph Cohen

Seconded: Haynes Miller, Paul Goerss

Received: 18 November 2008

Revised: 11 December 2009 